\title{
Data sharing for prevention: a case study in the development of a comprehensive emergency department injury surveillance system and its use in preventing violence and alcohol-related harms
}

\author{
Z ra Quigg, Karen Hughes, Mark A Bellis
}

Centre for Public Health, Liverpool John Moores University, Liverpool, UK

\section{Correspondence to} Zara Quigg, Centre for Public Health, Liverpool John Moores University, 15-21 Webster Street, Liverpool L3 2ET, UK z.a.quigg@ljmu.ac.uk

Accepted 28 November 2011 Published Online First 31 December 2011

\section{UNLCKED}

This paper is freely available online under the BMJ Journals unlocked scheme, see http:// injuryprevention.bmj.com/site/ about/unlocked.xhtml

\begin{abstract}
Objective To examine emergency department (ED) data sharing via a local injury surveillance system and assess its contribution to the prevention of violence and alcoholrelated harms.
\end{abstract}

Methods 6-year (2004-2010) exploratory study analysing injury attendances to one ED in the North West of England using descriptive and trend analyses.

Results Over the 6-year period, there were 242796 ED injury attendances, including 21683 for intentional injuries. Compared with unintentional injury patients, intentional injury patients were more likely to be men, aged 18-34 years, live in the most deprived communities, have attended the ED at night/weekends, have been injured in a public place and have consumed alcohol prior to the injury. Detailed data collected on alcohol and violence-related ED attendances were shared with local partners to monitor local trends and inform prevention activity including targeted policing and licensing enforcement. Over the 6-year period, intentional ED injury attendances decreased by $35.6 \%$ and alcoholrelated assault attendances decreased by $30.3 \%$.

Conclusions The collection of additional ED data on assault details and alcohol use prior to injury, and its integration into multi-agency policy and practice, played an important role in driving local violence prevention activity. Further research is needed to assess the direct contribution ED data sharing makes to reductions in violence.

\section{INTRODUCTION}

The WHO promotes the sharing and use of emergency department (ED) data as a major component in the public health approach to prevent injuries. ${ }^{1-3}$ Routine recording of attendance cause and patient demographics means ED data can be used to monitor injuries, identify 'at risk groups and communities' for targeting appropriate prevention activity and evaluate its impacts. Injury surveillance systems (ISSs) using data captured in EDs have been successfully established in both high and middle income countries. ${ }^{4-9}$ However, in many countries the use of ED data for injury prevention remains underdeveloped. ${ }^{8} 10$

In the UK, a range of ED-based ISSs have been established to inform injury prevention. With some exceptions (eg, All Wales ISS ${ }^{11}$ ), most have focused on specific injury types, ${ }^{12}$ injury severity ${ }^{13}$ or age groups. ${ }^{14}$ In England, a national commissioning dataset $(\mathrm{NCD})^{15}$ has been established that requires all EDs to collect a standard set of data on individual attendances, including injury group and location, patient demographics, attendance time and date, and arrival, referral and disposal method. These data were first published in 2009 and are updated annually. However, data are currently incomplete, of poor quality and not available at a suitable level for use in local injury prevention work. ${ }^{16}$ There is growing recognition across the UK of the utility of ED data in injury prevention. In particular, the government is promoting the collection of detailed data on violence and its sharing with police and other agencies to target violence prevention. ${ }^{17}$ In 2009, the College of Emergency Medicine published guidelines for EDs to support information sharing to reduce violence. ${ }^{18}$ Despite the increasing support for ED data sharing, local partners have struggled to access data. ${ }^{19}$ Factors including variations in data collection methods and information systems between EDs, poor understanding of what can be shared while maintaining patient confidentiality and a lack of incentives for health services to share data have hampered data sharing. ${ }^{19} 20$

In 2002, the Trauma and Injury Intelligence Group (TIIG) multi-agency ISS (box 1) was established to improve the availability and use of injury data in the English county of Merseyside, with a particular focus on ED data. Since its inception, TIIG has expanded to cover all 31 EDs (across 39 local authority areas) in the North West of England. TIIG offers a comprehensive ED ISS that covers all injury types, all population groups and operates flexibly to meet the needs of local partners. In this article, we describe the establishment of TIIG in Wirral local authority area. Wirral's single ED (Arrowe Park Hospital, Wirral NHS Trust) was one of the first to join the TIIG ISS and has consistently provided data since 2003/2004. The geographical location of the ED on the Wirral peninsula means that the majority of attendees (89.9\% in 2009/ 2010) are residents of Wirral local authority, which includes some of the most and least deprived communities in England. ${ }^{23}$ Continued ED data collection and sharing has provided partners with information to develop, target and implement injury prevention activities. In this study, we analyse ED data to identify the nature of injury presentations and trends in intentional and unintentional injury presentations over a 6-year period. We then assess the contribution of $\mathrm{ED}$ data sharing to local violence and alcohol-related harm prevention activity. 


\section{Box 1 The Trauma and Injury Intelligence Group (TIIG)}

TIIG was established in a county in the North West of England in 2001. The remit of the group (including representatives from health, criminal justice, the fire service and academia) was to establish the burden of injuries among the local population, recommend evidenced-based interventions to prevent injury and support the local implementation of such initiatives. A review of injury data sources indicated a lack of locally available data. ${ }^{21}$ Consequently, in 2002, TIIG secured funding to develop a population-based injury surveillance system (ISS). The funding enabled the employment of a dedicated TIIG officer to develop and manage the ISS, based at Liverpool John Moores University. Partners who were collecting electronic injury data on a routine basis were asked, and agreed to participate in the ISS. These included six emergency departments (EDs), the fire service and ambulance service. Data sharing and dissemination protocols were established and signed. ${ }^{22}$ In addition, hospital episodes and mortality data were accessed from pre-existing injury data sources (ie, North West Public Health Observatory; http://www. nwpho.org.uk). Data needs, reporting styles and schedules were determined through discussion with local partners. Reports have included monthly reports covering all ED injury attendances, more detailed reports on alcohol and violence (eg, bi-weekly reports for police) and annual reports on key injury issues, including childhood injuries, falls and violence. Since $2003 / 2004$, partners have also made more than 100 requests for ad hoc data analyses via the TIIG website (http://www.tiig.info) to support specific interventions.

\section{METHODS}

ED data sharing was established through a series of meetings between the TIIG and ED personnel including the ED manager, information manager and data confidentiality lead (Caldicott guardian $\left.{ }^{24}\right)$. Discussions focused on data sharing legislation, patient confidentiality, data availability/usage, the implications of involvement for ED practice and how additional demands on staff could be minimised. Data sharing protocols were established allowing pseudo-anonymised (eg, age opposed to date of birth) data to be shared with TIIG on a monthly basis via secure email. Initial data sharing commenced in 2002 and included data fields from the $\mathrm{NCD}^{15}$ and other injury-related data items already collected by the ED (including categories of injury types not recorded through the NCD). Data covered all ED patients entered onto their electronic patient administration system, and included data collected through a series of routine questions asked during the booking in process (by receptionists), at triage (by nurses) and during treatment and discharge (by consultants).

Consultation with local partners (eg, public health professionals, police) identified violence, alcohol-related injuries and injuries in the home as key prevention priorities. Thus, the hospital removed fields that were not being used for administrative, clinical or strategic purposes, and added data items (in 2003/2004) to support local priorities. These were for collection by ED receptionists and included: for all attendances, whether alcohol had been consumed in the $3 \mathrm{~h}$ preceding the incident; and for assault patients, the location of assault and number of attackers. Reception staff training for collecting the additional data and improving overall data collection quality was provided by the TIIG officer, based at Liverpool John Moores University.
Data quality was monitored by the ED data quality officer. Full data sharing commenced in 2003/2004, with the TIIG officer cleaning and analysing the data and providing routine reports, themed injury analyses and ad hoc data requests to partners. All partners accessing data throughout the 6 years were asked to provide details of how they intended to use the data, its purpose and the perceived impact of data usage. In addition, local partners provided verbal feedback to the ED and the TIIG staff in ED staff meetings. Further information on data usage was obtained through partner surveys, which aimed to assess data accessibility, usage and forthcoming injury prevention priorities.

For this study, ED data covering six financial years (April 2004-March 2010) were extracted from the TIIG ISS. Variables included in the analyses were age, sex, injury group, incident location, attendance time/date, whether the patient had consumed alcohol within $3 \mathrm{~h}$ preceding the incident and disposal method. Injuries were categorised into intentional (ie, violence and self-harm) and unintentional. In order to assign individuals with a measure of deprivation, their postcode of residence was mapped to a lower super output area (a geographical area (population mean=1500) used to standardise reporting of small area statistics in England and Wales) ${ }^{25}$ and linked to the Index of Multiple Deprivation 2007 (a composite measure of deprivation combining economic, social and housing data ${ }^{23}$ ). Analyses examined trends in ED attendances for intentional and unintentional injury using descriptive statistics and $\chi^{2}$ tests (including $\chi^{2}$ for a trend). Data were analysed using SPSS V.17.

\section{RESULTS}

Over the 6 years there were $242796 \mathrm{ED}$ injury attendances (accounting for $44.5 \%$ of all attendances). Most injury groups shared with TIIG are not recorded in the NCD (table 1). For example, falls are not recorded as a separate injury category in

Table 1 Incident type of emergency department attendances by the NCD and TIIG recoded dataset, 2009/2010

\begin{tabular}{llrr}
\hline Injury category & Incident type & NCD & TIIG dataset \\
\hline Intentional & Assault & 2035 & 2035 \\
& Firearm & - & 2 \\
Unintentional & Deliberate self-harm & 662 & 662 \\
& Road traffic collision & 3497 & 3497 \\
& Sports injury & 1354 & 1354 \\
& Fall & - & 15910 \\
& Struck (blunt object) & - & 4926 \\
& Wound/cut & - & 2649 \\
& Burn/scold & - & 367 \\
& Bite & - & 378 \\
& Ingestion & - & 338 \\
& Inhalation & - & 56 \\
& Non-fire burn/scold & - & 43 \\
& Electrical & - & 14 \\
Drown/immersion & - & 11 \\
Total injury attendances & Other injury (including fireworks) & 15275 & 3455 \\
Total attendances & 22823 & 35697 \\
\hline
\end{tabular}

The emergency department collects additional data on injury type (eg, fall/burn) in a separate field to the NCD injury group field. Here, data collection methods mean that an attendance can be recorded as 'other injury' within the NCD injury grouping yet still be identified as a fall- or burn-related injury, for example, in the addition field. TIIG combine fields to provide partners with the most detailed and useful data on injury type, with each attendee categorised into one injury group.

NCD, national commissioning dataset; TIIG, Trauma and Injury Intelligence Group. 
the NCD, instead mostly being coded as 'other injury' or 'medical' attendances; the TIIG dataset identified 15910 fallrelated ED attendances in 2009/2010, accounting for $17.7 \%$ of all attendances and $44.6 \%$ of injury attendances. Overall, the TIIG identified 12874 more injury attendances in 2009/2010 than the NCD. Further, $66.9 \%$ of injury attendances recorded through the NCD were categorised as 'other injury', accounting for one in five (17.0\%) ED attendances. In comparison, $9.6 \%$ of the injury attendances recorded through TIIG were recorded as 'other injury', accounting for $3.8 \%$ of all $\mathrm{ED}$ attendances.

Table 2 shows the demography and circumstances of ED injury attendances by year. Across the 6 years, the majority (91.1\%) of presentations were for unintentional injuries (eg, $44.9 \%$ fall-related, $15.8 \%$ struck by an object, $10.4 \%$ road traffic collision). Assaults accounted for $70.2 \%$ of intentional injury attendances and deliberate self-harm $29.6 \%$; $<1 \%$ were recorded as gunshot wounds or stabbings. There were significant demographic (ie, age, gender, area of residence) and circumstantial (ie, injury location, attendance time/date) differences between unintentional and intentional injury patients. For example, compared with unintentional injury, intentional injury attendees were more likely to be men $(62.0 \%)$, aged $18-34$ years $(41.3 \%)$, live in the most deprived communities $(58.8 \%)$, have been injured in a public place $(58.3 \%)$ and have consumed alcohol within $3 \mathrm{~h}$ prior to injury (47.2\%). Further, they were more likely to attend the ED at night (18:00-05:59: 63.7\% cf. $34.0 \% ; \mathrm{p}<0.001$ ) and at weekends (Friday-Sunday: $53.6 \%$ cf. $41.6 \% ; \mathrm{p}<0.001)$. Compared with unintentional injury attendances, a higher proportion of intentional injury attendances were admitted into hospital for further treatment/monitoring (32.2\% cf. $10.7 \%$; $\mathrm{p}<0.001)$.

Over the 6 years, the majority $(96.4 \%)$ of injury attendees were asked if they had consumed alcohol within $3 \mathrm{~h}$ prior to their injury. Nearly half $(47.2 \%)$ of intentional injury and $4.9 \%$ of unintentional injury patients reported drinking alcohol $(p<0.001)$. One-third $(33.0 \%)$ of alcohol-related injury attendances were recorded as an assault, $27.9 \%$ a fall and $15.6 \%$ deliberate self-harm. Alcohol-related ED attendees were more likely $(p<0.001)$ to be men, aged 18-34 years, reside in the most deprived communities and attend the ED at night/weekends. Patients who reported having consumed alcohol prior to injury were more likely to be admitted to hospital than those who had not consumed alcohol (intentional injury: $37.5 \%$ cf. 19.7\%, $\mathrm{p}<0.001$; unintentional injury: $24.0 \%$ cf. $9.6 \%, \mathrm{p}<0.001$ ).

$\chi^{2}$ for a trend analyses showed significant decreases in both intentional $(35.6 \% ; \mathrm{p}<0.001)$ and unintentional $(11.5 \%$; $p<0.001$ ) injury attendances. For unintentional injuries, decreases were seen in all age groups except the $0-4$ years and $65+$ years age groups. For intentional injuries, all age groups decreased with the largest decreases seen in the 5-17 years $(49.1 \%)$ and $65+$ years $(43.5 \%)$ age groups. The proportion of intentional injury attendances that were admitted to hospital decreased $(5.3 \% ; \mathrm{p}<0.001)$, while the proportion of unintentional injury attendances that were admitted increased $(1.3 \%$; $\mathrm{p}<0.001)$. There were decreases in alcohol-related injury attendances (assaults, 30.3\%; falls, 23.4\%; deliberate self-harm, $56.9 \%)$. There was a decrease in the number of falls recorded as alcohol-related, compared with non alcohol-related falls $(23.4 \%$ cf. $0.8 \% ; \mathrm{p}<0.001)$.

\section{DISCUSSION}

This study has described the establishment of ED data sharing in a local municipality in the UK, as part of the development of a broader ISS. Injury surveillance is widely recognised to be

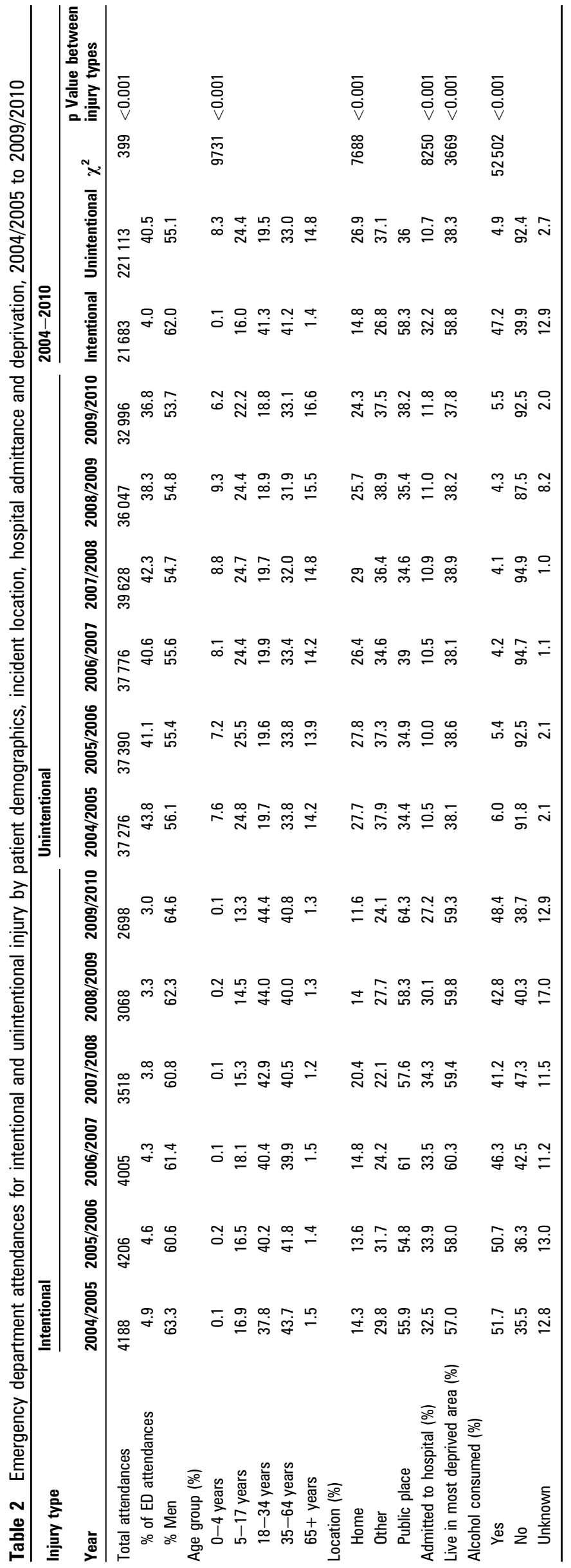




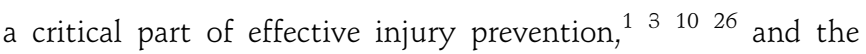
collection and sharing of local ED data can enable the identification of the burden of injuries in a given area, their nature and those groups most at risk. ${ }^{1} 9{ }^{10}$ For example, the TIIG ISS identified that one-fifth of all ED attendances were fall-related, which are recorded as 'other injury' in the national ED data recording system. Yet ED data have some inherent flaws. They are rarely population based and can be biased by factors including site location. ${ }^{27}$ Further, the consistency of data collection for surveillance purposes can vary. In our study, for instance, consistency in the collection of alcohol consumption data from injury patients varied over time, although regular staff training and feedback on data usage resulted in information being collected for over $80 \%$ of all injury patients each year. Further, patients may not disclose the cause of their injury, resulting in under-recording of some injury types. For example, one study found that many victims of domestic violence would not report violence as the cause of their injury, while others would only report their abuse if asked directly, preferably by a physician. ${ }^{28}$ Despite these issues, our study has shown that ED data sharing can be achieved and barriers to accessing and utilising local data can be overcome. ${ }^{10} 19$

Overall, analyses of ED data show there have been reductions in both intentional and unintentional injury attendances over the 6 years. Intentional injury presentations to the ED decreased by $35.6 \%$ and unintentional injuries by $11.5 \%$. A significant decrease was also seen in the proportion of intentional (but not unintentional) injury attendances admitted to hospital for further treatment, potentially suggesting a decrease in the severity of assaults occurring in the municipality. The major driver for ED data sharing across this municipality (and across England) has been the prevention of violence and alcohol-related harms, and this may help explain the greater reductions seen in intentional injuries compared with unintentional injuries. Although it is not possible to attribute reductions in violence to ED data sharing, the data have been used by a range of agencies to target prevention work (see figure 1), and we can identify the role that data sharing played in focusing multi-agency resources towards violence and alcohol-related harm reduction and in monitoring their impacts.
ED data have been used to inform the development of local multi-agency strategies for preventing violence and alcoholrelated harm across the municipality ${ }^{29}{ }^{30}$; set a community safety target to reduce alcohol-related ED assault attendances by 15\% (2004/2005-2007/2008); and develop, target and evaluate activities (figure 2). ${ }^{31}$ To facilitate data usage, bi-monthly multiagency meetings (including TIIG, health and community safety leads) have been held during which data and interventions were discussed. Prevention activities have included enforcement work targeted at drinking establishments identified as the locations of assaults resulting in ED treatment. Such enforcement has included police and licensing officer venue visits to check compliance with UK licensing laws and provide support in preventing violence and alcohol-related harm within and around the venue. In 2007/2008, data on glass-related injury attendances were used to encourage licensed premises to use polycarbonate (plastic) glassware during peak times for violence. During 2007/ 2008, the ED recorded eight glass-related incidents, compared with 22 in 2006/2007. Between 2004/2005 and 2007/2008, the number of ED attendances due to alcohol-related assault declined beyond the target $15 \%$ and have decreased by $34.3 \%$ since TIIG data sharing commenced (assault data from 59 EDs in England and Wales also show reductions although to a much lesser extent ${ }^{12}$ ). The success has largely been facilitated by strong partnership working between agencies and a commitment to use an evidenced-based approach to prevention.

It is widely acknowledged that many incidents of violence are not reported to police, yet those that result in injury can often require health treatment through an ED. ${ }^{9}{ }^{32}$ In Wirral (2009/ $2010)$, the police were not aware of $25 \%$ of ED reported assault incidents. In England, increasing concerns about serious youth violence have led the government to prioritise and now mandate the collection and sharing of additional ED data on violence (ie, assault location, time and weapon ${ }^{17}{ }^{18}$ ). As shown in Wirral, and other areas across England and Wales, ${ }^{33} 34$ ED data can support the targeting of interventions in areas and drinking premises where violence occurs. Florence et $a l^{34}$ found that the use of ED data to inform targeted policing contributed to a reduction in violence-related hospital admissions, yet an increase in minor assaults reported by police. Our experience has identified
Figure 1 Examples of TIIG data sharing pathways and local partner data usage. ISS, injury surveillance system; TIIG, Trauma and Injury Intelligence Group.

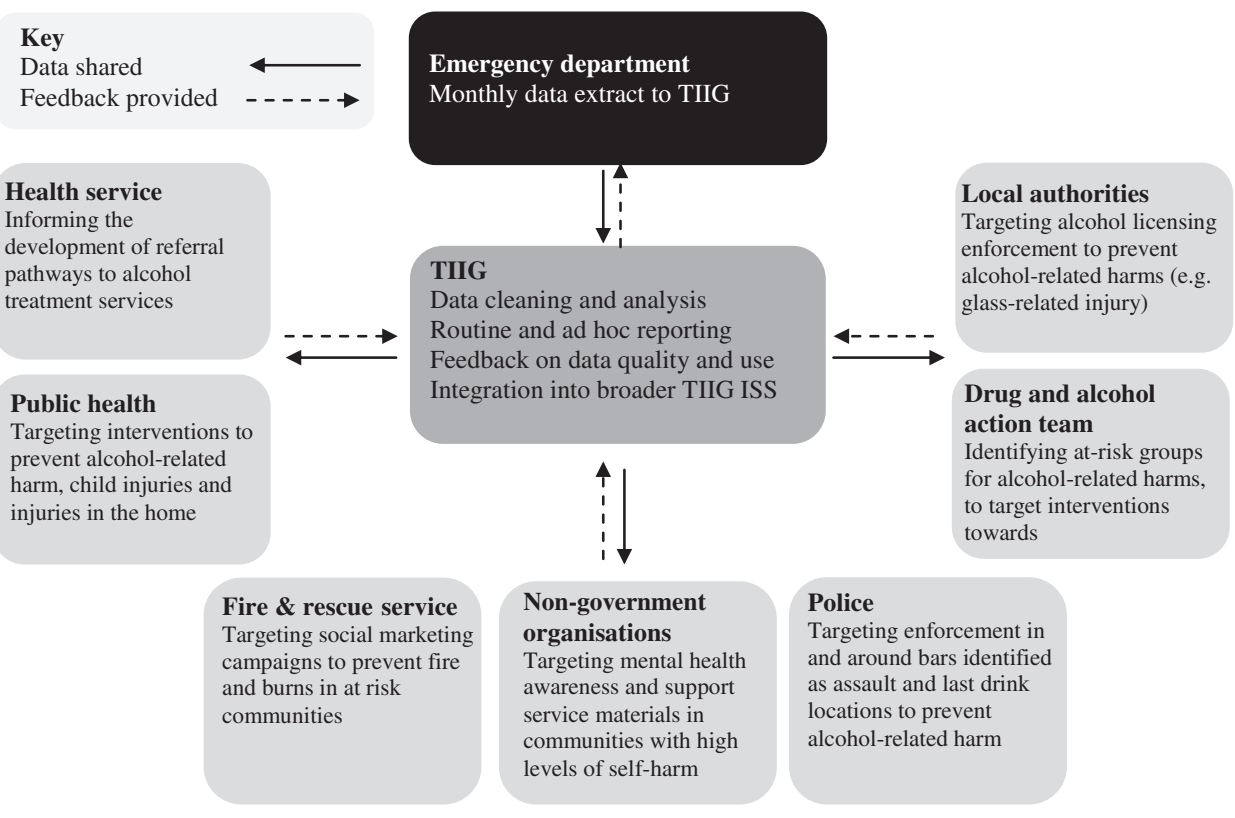




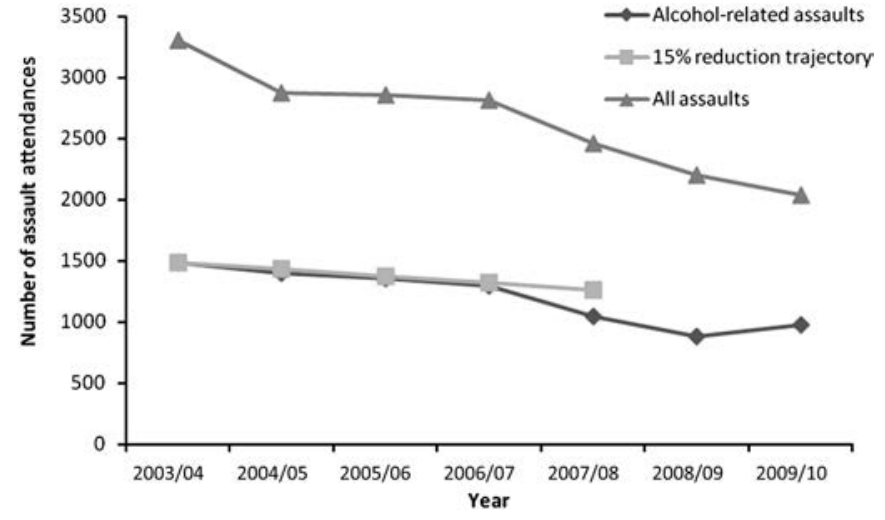

Figure 2 Emergency department attendances for assaults, alcoholrelated assaults and community safety target reduction trajectory, by year.

a number of elements that have facilitated ED data sharing and use that are likely to be transferable to other parts of the UK and also internationally. ${ }^{8} 10$ These include gaining support for data sharing among all ED staff (including administrative/information technology staff who collect/manage the data); forging partnerships between EDs and injury prevention partners; developing data sharing protocols; linking additional data items to existing electronic ED systems; ensuring routine data usage by local partners; and providing ED staff with feedback on how their data are used in practice, the results this achieves and data quality.

Limiting ED data sharing to the location of assault focuses resources on policing and enforcement in areas where assaults occur. TIIG data identify strong links between assaults and deprivation, showing that while violence peaks in town and city centres at weekend nights the majority of assault patients live in the most deprived communities. Emergency hospital admissions for violence in England also show strong relationships with deprivation, with those from the most deprived communities being over five times more likely to require admission for assault than those from the least deprived communities. ${ }^{35}$ Here, the greatest rate ratios between the least and most deprived areas are seen in childhood and later adulthood. Thus, while targeting police resources in nightlife environments can reduce violence occurring in such locations, the tendencies that lead to violence in young adults are likely established far earlier in life as they grow up in violent communities. ED data on assault victims' area of residence should be used alongside that of the location of assault to implement broader violence prevention strategies.

This is one of the first studies in the UK to examine the role of ED data sharing in violence prevention. ${ }^{33}$ Our findings support associations between data sharing and reductions in violence identified by Florence et al. ${ }^{34}$ However, whether data sharing is a critical component in a multi-agency response to tackling violence or symbolic of strong multi-agency responses being in place has yet to be established. With ED data sharing now being promoted, initiated and implemented at various levels throughout the UK, the current heterogeneity of approaches is likely to provide sufficient variation for a broad ecological analysis of factors associated with reductions in violence. Elsewhere, a prospective approach to identifying any causal relationships between data sharing and violence prevention could use a study design where similar areas without data sharing are matched, and data sharing is deliberately introduced into one set of cases for comparison with control areas. Such studies would need to take into account a variety of factors

\section{What is already known on the subject}

Emergency department (ED) data can play a key role in developing a public health approach to injury prevention.

- The sharing of enhanced ED data to inform local violence prevention is a major government priority in the UK and elsewhere, yet many areas struggle to establish such data sharing.

- Key barriers to collecting and sharing ED data for use in injury prevention include a lack of understanding of what data can be shared and constraints with information technology.

\section{What this study adds}

- We identify how an effective ED data sharing system has been established as part of a comprehensive injury surveillance system.

- Reductions in violence were seen over the 6-year period which included the systematic integration of ED data on violence (including alcohol involvement and the nature of assault) into local violence prevention planning, activity and monitoring.

- The identification of associations between ED data sharing, multi-agency prevention activity and violence reduction support the need for more robust research to measure the specific role of ED data in violence prevention.

including socio-demographics, alcohol outlets density and approaches to violence prevention being implemented locally.

\section{CONCLUSION}

The establishment and use of the TIIG ISS in one municipality in England demonstrates that comprehensive data collection and sharing between EDs and multi-agency partnerships can be achieved and sustained, and can play a key role in supporting injury prevention. Focusing on intentional injuries, our study has demonstrated how additional ED data collection on alcohol and violence-related attendances, and the development of a multi-agency partnership to promote the use of ED data at a local level, has supported targeted interventions, such as policing and licensing enforcement. ED data provide vital intelligence on patient demographics that could also usefully inform broader violence prevention work towards individuals and communities most at risk. While this study shows significant reductions in intentional and alcohol-related assault attendances to the $\mathrm{ED}$, further research is required to identify the specific contribution of data sharing to violence prevention.

Acknowledgements We would like to thank Wirral University Teaching Hospital NHS Foundation Trust, particularly Christine Kennedy and reception staff working within the ED who collect the additional data, for their support and dedication. Finally we would like to thank the local Primary Care Trust and Community Safety Partnership, and staff working at the Centre for Public Health, Liverpool John Moores University, particularly Daniel Hungerford, for supporting the development and ongoing implementation of the TIIG ISS.

Funding The Trauma and Injury Intelligence Group (TIIG) injury surveillance system (ISS) in Wirral is funded by Wirral Primary Care Trust.

Competing interests None. 
Contributors $\mathrm{ZO}$ analysed the data and produced the manuscript. $\mathrm{KH}$ and $\mathrm{MAB}$ assisted in the production of the manuscript. All authors read and reviewed the final manuscript.

Provenance and peer review Not commissioned; externally peer reviewed.

Data sharing statement The emergency department data were obtained from Wirral NHS Trust. All data shared were depersonalised and conform to the UK Data Protection Act 1998 and relevant government data sharing guidelines.

\section{REFERENCES}

. Holder Y, Peden M, Krug E, et al. Injury Surveillance Guidelines. Geneva: World Health Organisation, 2001.

2. Butchart A, Brown D, Khanh-huynh A, et al. Manual for estimating the economic costs of injuries due to interpersonal and self-directed violence. Geneva: World Health Organisation and Department of Health and Human Services, Centers for Disease Control and Prevention, 2008.

3. Peden M, Scurfield R, Sleet D, et al. World Report on Road Traffic Injury Prevention. Geneva: World Health Organisation, 2004

4. Mackenzie SG, Barry Pless I. CHIRPP: Canada's principal injury surveillance program. Inj Prev 1999;5:208-13.

5. Quinlan KP, Thompson MP, Annest JL, et al. Expanding the National Electronic Injury Surveillance System to monitor all nonfatal injuries treated in US hospital emergency departments. Annals Emerg Med 1999:34:637-45.

6. Zuckerbraun NS, Powell EC, Sheehan KM, et al. Community childhood injury surveillance: an emergency department-based model. Pediatr Emerg Care 2004;20:361-6.

7. Sanchez CA, Thomas KE, Malilay J, et al. Nonfatal natural and environmental injuries treated in emergency departments, United States, 2001-2004. Fam Community Health 2010:33:3-10.

8. Espitia-Hardeman V, Borse NN, Dellinger AM, et al. The burden of childhood injuries and evidence based strategies developed using the injury surveillance system in Pasto, Colombia. Inj Prev 2011:17(Suppl 1):i38-44.

9. Faergemann C, Lauritsen JM, Brink 0 , et al. Trends in deliberate interpersonal violence in the Odense Municipality, Denmark 1991-2002. The Odense study on deliberate interpersonal violence. J Forensic Leg Med 2007;14:20-6.

10. Nilson $\mathbf{P}$, Bourne $M$, Coggan C. Using local injury surveillance for community-based injury prevention: an analysis of Scandinavian WHO safe community and Canadian safe community foundation programmes. Int J Inj Control Safe Promot 2007; 14:35-43.

11. Collaboration for Accident Prevention and Injury Control (CAPIC). Accident and Emergency Department Data: All Wales Injury Surveillance Systems (AWISS). http://www.capic.org.uk/ (accessed 1 Feb 2011).

12. Sivarajasingam V, Wells JP, Moore S, et al. Violence in England and Wales in 2010. An Accident and Emergency Perspective. Cardiff: Cardiff University, 2008

13. Trauma Audit Research Network (TARN). https://www.tarn.ac.uk (accessed 1 Jul 11).

14. Shipton D, Stone DH. The Yorkhill CHIRPP story: a qualitative evaluation of 10 years of injury surveillance at a Scottish children's hospital. Inj Prev 2008;14:245-9.

15. Connecting for Health. Commissioning Dataset Overview. http://www. datadictionary.nhs.uk/web_site_content/navigation/ commissioning data sets menu.asp (accessed 1 Oct 2010).
16. Health and Social Care Information Centre. Accident and Emergency Attendances in England (Experimental Statistics) 2008-09. London: The NHS Information Centre, 2010.

17. HM Government. The Coalition: Our Programme for Action. London: Cabinet Office 2010.

18. Boyle A, Shepherd J, Sheehan D. Guideline for Information Sharing to Reduce Community Violence. London: College of Emergency Medicine, 2009.

19. Davidson T, van Staden L, Nicholas S, et al. Process Evaluation of Data Sharing between Emergency Departments and Community Safety Partnerships in the South East. London, Home Office, 2010.

20. Boyle A, Snelling K. Information sharing to reduce community violence: culture shift and incentives are needed. BMJ 2011:343:d4452.

21. Fell R, Tocque K, Hockey G, et al. Accident, Injury and Trauma Information and Intelligence Initiatives in Merseyside, the North West and Nationwide. Liverpool: North West Public Health Observatory, Centre for Public Health, Liverpool John Moores University, 2002.

22. Trauma and Injury Intelligence Group. Information Sharing Protocol Governing the Exchange of Data for the Trauma and Injury Intelligence Group. Liverpool: Centre for Public Health, John Moores University, 2010.

23. HM Government. Indices of Deprivation 2007 for Super Output Areas. http://data. gov.uk/dataset/index_of_multiple_deprivation_imd_2007 (accessed 9 May 2011).

24. Department of Health. Caldicott Guardian Manual 2010. London: Department of Health, 2010.

25. Office for National Statistics. Super Output Areas Explained (Online). http://www. neighbourhood.statistics.gov.uk/dissemination/Info.do?page=aboutneighbourhood/ geography/superoutputareas/soa-intro.htm (accessed 14 Jul 2011).

26. Sethi D, Racioppi F, Baumgarten I, et al. Injuries and Violence in Europe. Why They Matter and What Can Be Done. Rome: WHO Regional Office for Europe, 2006.

27. Stone DH, Morrison A, Smith GS. Emergency department injury surveillance systems: the best use of limited resources? Inj Prev 1999;5:166-7.

28. Hayden SR, Barton ED, Hayden M. Domestic violence in the emergency department: how do women prefer to disclose and discuss the issues? J Emerg Med 1997:4:447-51.

29. Birkenhead and Wallasey Primary Care Trust. Wirral Evening and Night Time Strategy 2004-2008. Wirral: Birkenhead and Wallasey Primary Care Trust, 2004.

30. Wirral DAAT. Wirral's Alcohol Harm Reduction Strategy 2007-2010. Wirral: Wirra DAAT, 2007. http://www.wirraldaat.org

31. Bellis MA, Anderson Z, Hughes K. Effects of the Alcohol Misuse Enforcement Campaigns and the Licensing Act 2003 on Violence. A Preliminary Assessment of Accident and Emergency Attendances in Wirral. Liverpool: Centre for Public Health, John Moores University, 2006. http://www.tiig.info

32. Flatley J, Kershaw C, Smith K, et al. Crime in England and Wales 2009/10. Findings from the British Crime Survey and Police Recorded Crime. London: Home Office, 2010.

33. Dines C. Using A\&E data to prevent violence in communities. Nurs Times 2011:107:16-18.

34. Florence C, Shepherd H, Brennan I, et al. Effectiveness of anonymised information sharing and use in health service, police, and local government partnership for preventing violence related injury: experimental study and time series analysis. $B M$ 2011;342:d3313.

35. Bellis MA, Hughes $\mathrm{K}$, Wood $\mathrm{S}$, et al. National five-year examination of inequalities and trends in emergency hospital admission for violence across England. Inj Prev 2011:17:319-25

\section{Young Australian travellers make travel insurance claims}

Older Australian travellers pay higher travel insurance premiums even though it is those aged 23-30 who make the most claims mostly for damaged personal items, fractures or ligament injuries. Medical and dental claims were also high in this age group, compared with the 60-70 age group. Apparently, it is not the general health of travellers that is the issue but alcohol and mindless behaviour.

\section{The value of universal motorcycle helmet laws}

A new CDC study shows that universal helmet laws that require every motorcycle rider and passenger to wear a helmet whenever they ride increases helmet use and saves money. In 2010, cost savings in states with universal motorcycle helmet laws were nearly four times greater (per registered motorcycle) than in states without these comprehensive laws. Annual costs saved from helmet use ranged from a high of $\$ 394$ million in California (which has a universal helmet law) to a low of $\$ 2.6$ million in New Mexico (which has a partial law or a law requiring that only certain riders wear helmets). 